\title{
Cyclic quasi-contractions of Ćirić type in b-metric spaces
}

\author{
Fei He*, Xiao-Yue Zhao, Yu-Qi Sun \\ School of Mathematical Sciences, Inner Mongolia University, Hohhot 010021, China.
}

Communicated by R. Saadati

\begin{abstract}
In this paper, we give a negative answer to the open question raised by Radenovic et al. [S. Radenović, T. Došenović, T. A. Lampert, Z. Golubovíc, Appl. Math. Comput., 273 (2016), 155-164]. Namely, we give two examples which show that the set of fixed points for cyclic quasi-contractive mappings of Ćirić type may be empty. Then, by using a new lemma, we give some sufficient conditions for the existence of fixed point for cyclic and non-cyclic quasi-contractive mappings of Ćirić type in b-metric spaces. In particular, we show that the condition of Fatou property in the result of Amini-Harandi [A. Amini-Harandi, Fixed Point Theory, 15 (2014), 351-358] may be omitted. (C)2017 All rights reserved.
\end{abstract}

Keywords: b-metric space, fixed point, cyclic quasi-contractions of Ćirić type, Fatou property. 2010 MSC: 47H10, 54H25.

\section{Introduction and preliminaries}

In [4], Ćirić established a fixed point theorem for quasi-contractive mappings, which is one of the most general results in generalizations of classical Banach's contraction principle. Since then, many authors discussed and generalized Ćirić type fixed point theorem in various directions, see, for instance, [1, 7$10,13,18,19]$.

The concept of b-metric was introduced by Czerwik [6] (see also Bakhtin [3]) as a generalization of metric. Following this paper, a number of fixed point results in b-metric spaces were given, see $[1,2,11,12,14-17]$ and references therein. In particular, Amini-Harandi [1] established a Ćirić type fixed point result in b-metric spaces with Fatou property.

Definition $1.1([6])$. Let $X$ be a nonempty set and $s \geqslant 1$, a given real number. A mapping $d: X \times X \rightarrow[0, \infty)$ is called a $b$-metric on $X$, if it satisfies the following conditions:

(b1) $d(x, y)=0$, if and only if $x=y$;

(b2) $d(x, y)=d(y, x)$;

\footnotetext{
${ }^{*}$ Corresponding author

Email address: hefei@imu.edu.cn (Fei He)
}

doi:10.22436/jnsa.010.03.18 
(b3) $d(x, z) \leqslant s[d(x, y)+d(y, z)]$, for all $x, y, z \in X$.

In this case, the pair $(X, d)$ is called a b-metric space.

For more notions such as b-convergence, b-Cauchy sequence and b-completeness in b-metric spaces, the reader can refer to $[6,12]$.

Very recently, Radenović et al. [16] obtained some equivalences between cyclic contractions and noncyclic contractions in b-metric spaces. Moreover, they proposed an open question as follows.

Question 1.2. Prove or disprove the following:

Let $\left\{A_{i}\right\}_{i=1}^{p}$, where $p$ is a positive integer, be nonempty closed subsets of a b-complete b-metric space $(X, d)$ with $s \geqslant 1$ and $\mathrm{T}: \cup_{i=1}^{p} A_{i} \rightarrow \cup_{i=1}^{p} A_{i}$ satisfies the following conditions (where $A_{p+1}=A_{1}$ )

(i) $\mathrm{T}\left(\mathrm{A}_{\mathrm{i}}\right) \subseteq A_{i+1}$ for $1 \leqslant i \leqslant p$;

(ii) there exists $\lambda \in\left[0, \frac{1}{\mathrm{~s}}\right)$ such that

$$
d(T x, T y) \leqslant \lambda \max \{d(x, y), d(x, T x), d(y, T y), d(x, T y), d(y, T x)\},
$$

for all $x \in A_{i}, y \in A_{i+1}, 1 \leqslant i \leqslant p$.

If $(\mathrm{X}, \mathrm{d})$ has a Fatou's property, then $\mathrm{T}$ has a unique fixed point.

In this paper, inspired by the counterexample given by Ćirić in [5, Example 4], we give two examples which show that the set of fixed points for cyclic mappings of Ćirić type may be empty in metric spaces, as well as in b-metric spaces with Fatou property. From this, we give an negative answer to Question 1.2. Then, in order to consider the existence of fixed point for cyclic quasi-contractive mappings of Ćirić type, we give a new lemma. Using this lemma, we establish some fixed point results for cyclic and noncyclic quasi-contractions of Ćirić type in b-metric spaces. Particularly, we see that the condition of Fatou property in the result of Amini-Harandi [1] may be removed.

\section{Main results}

We begin with the following examples.

Example 2.1. Let $X$ be the Euclidean space $\mathbb{R}^{3}$ with the usual metric,

$$
A=\{(0,0,0),(4,0,0)\} \quad \text { and } \quad B=\{(2,2,0),(2,-2,1)\} .
$$

Set

$$
x=(0,0,0), \quad y=(4,0,0), \quad u=(2,2,0), \quad v=(2,-2,1) .
$$

Then

$$
\begin{array}{rrrl}
d(x, y) & =4, & d(x, u)=2 \sqrt{2}, & d(x, v)=3, \\
d(y, u)=2 \sqrt{2}, & d(y, v)=3, & d(u, v)=\sqrt{17}
\end{array}
$$

Define $T: A \cup B \rightarrow A \cup B$ by

$$
\mathrm{T} x=u, \quad \mathrm{Ty}=v, \quad \mathrm{Tu}=\mathrm{y} \quad \text { and } \quad \mathrm{T} v=x .
$$

According to the definitions of the sets $A, B$ and the mapping $T$, it follows that $T A \subseteq B$ and $T B \subseteq A$. Further, we have

$$
d(T x, T u)=d(u, y)=2 \sqrt{2}<d(x, T u)=d(x, y)=4,
$$




$$
\begin{aligned}
& d(T x, T v)=d(u, x)=2 \sqrt{2}<d(v, T x)=d(v, u)=\sqrt{17} \\
& d(T y, T u)=d(v, y)=3<d(u, T y)=d(u, v)=\sqrt{17} \\
& d(T y, T v)=d(v, x)=3<d(y, T v)=d(y, x)=4
\end{aligned}
$$

Thus T satisfies all conditions of Question 1.2, where $s=1$ and $\lambda=\frac{3}{4}$, but T does not have any fixed point in $A \cap B$.

Example 2.2. Let $X=\mathbb{R}^{3}$ and $\|\cdot\|$ the usual norm on $X$. Define $d: X \times X \rightarrow[0, \infty)$ by

$$
d(x, y)=\|x-y\|^{\frac{3}{2}} .
$$

We can deduce that $(X, d)$ is a b-metric space with $s=\sqrt{2}$. In fact, since $\varphi(t)=t^{\frac{3}{2}}(t \geqslant 0)$ is a concave function, we have, for all $x, y, z \in X$,

$$
\begin{aligned}
d(x, y) & =\|x-y\|^{\frac{3}{2}} \leqslant(\|x-z\|+\|z-y\|)^{\frac{3}{2}} \\
& =2^{\frac{3}{2}} \cdot\left(\frac{\|x-z\|+\|z-y\|}{2}\right)^{\frac{3}{2}} \\
& \leqslant 2^{\frac{3}{2}} \cdot \frac{\|x-z\|^{\frac{3}{2}}+\|z-y\|^{\frac{3}{2}}}{2} \\
& =\sqrt{2}[d(x, z)+d(z, y)] .
\end{aligned}
$$

Let $A=\{x, y\}$ and $B=\{u, v\}$, where

$$
x=(0,0,0), \quad y=(4,0,0), \quad u=(2,2,0), \quad v=(2,-2,1) .
$$

Define $T: A \cup B \rightarrow A \cup B$ by

$$
\mathrm{Tx}=\mathrm{u}, \quad \mathrm{Ty}=v, \mathrm{Tu}=\mathrm{y} \quad \text { and } \mathrm{T} v=x .
$$

According to the definitions of the sets $A, B$ and the mapping $T$, it follows that $T A \subseteq B$ and $T B \subseteq A$. Clearly, $(X, d)$ has a Fatou property because $d$ is a continuous mapping. Further, we have

$$
\begin{aligned}
& d(T x, T u)=d(u, y)=(2 \sqrt{2})^{\frac{3}{2}} \leqslant \frac{1}{\sqrt{2}} \cdot \frac{15}{16} d(x, T u)=\frac{1}{\sqrt{2}} \cdot \frac{15}{16} \cdot 4^{\frac{3}{2}}, \\
& d(T x, T v)=d(u, x)=(2 \sqrt{2})^{\frac{3}{2}} \leqslant \frac{1}{\sqrt{2}} \cdot \frac{15}{16} d(v, T x)=\frac{1}{\sqrt{2}} \cdot \frac{15}{16} \cdot \sqrt{17^{\frac{3}{2}}}, \\
& d(T y, T u)=d(v, y)=3^{\frac{3}{2}} \leqslant \frac{1}{\sqrt{2}} \cdot \frac{15}{16} d(u, T y)=\frac{1}{\sqrt{2}} \cdot \frac{15}{16} \cdot \sqrt{17^{\frac{3}{2}}}, \\
& d(T y, T v)=d(v, x)=3^{\frac{3}{2}} \leqslant \frac{1}{\sqrt{2}} \cdot \frac{15}{16} d(y, T v)=\frac{1}{\sqrt{2}} \cdot \frac{15}{16} \cdot 4^{\frac{3}{2}} .
\end{aligned}
$$

Thus T satisfies all conditions of Question 1.2, where $s=\sqrt{2}$ and $\lambda=\frac{15}{16 \sqrt{2}}$, but $T$ does not have any fixed point in $A \cap B$.

Remark 2.3. From Example 2.1 and Example 2.2, we see that the set of fixed points for cyclic quasicontractions of Ćirić type may be empty. This means that the open question raised by Radenović et al. (that is, Question 1.2) has been answered.

Next we give some sufficient condition for the existence and uniqueness of fixed point for Ćirić type cyclic quasi-contractive mappings. To this end, we need the following lemma.

Lemma 2.4. Let $(X, d)$ be a b-complete b-metric space with $s \geqslant 1$ and $\left\{A_{i}\right\}_{i=1}^{p}$ nonempty closed subsets of $X$. Suppose that $\mathrm{T}: \cup_{i=1}^{p} A_{i} \rightarrow \cup_{i=1}^{p} A_{i}$ satisfies the following conditions (where $A_{p+1}=A_{1}$ ) 
(i) $T\left(A_{i}\right) \subseteq A_{i+1}$ for $1 \leqslant i \leqslant p$;

(ii) there exists $\lambda \in\left[0, \frac{1}{s}\right)$ such that

$$
d(T x, T y) \leqslant \lambda \max \{d(x, y), d(x, T x), d(y, T y), d(x, T y), d(y, T x)\},
$$

for all $x \in A_{i}, y \in A_{i+1}, 1 \leqslant i \leqslant p$.

If the following condition (F) holds,

(F) there exists $x_{0} \in \cup_{i=1}^{p} A_{i}$ such that

$$
\lim _{n \rightarrow \infty} d\left(x_{n}, x_{n+1}\right)=0
$$

where $x_{n}=T x_{n-1}, n=1,2, \cdots$,

then $\mathrm{T}$ has a unique fixed point.

Proof. By the condition (F), there exists $x_{0} \in \cup_{i=1}^{p} A_{i}$ such that

$$
\lim _{n \rightarrow \infty} d\left(x_{n}, x_{n+1}\right)=0,
$$

where $x_{n}=T x_{n-1}, n=1,2, \cdots$. Denote

$$
\begin{aligned}
D_{n} & =\max \left\{d\left(x_{i}, x_{j}\right): 0 \leqslant i, j \leqslant n \text { and } i, j \in \mathbb{N}\right\}, \\
D_{\infty} & =\sup \left\{d\left(x_{i}, x_{j}\right): i, j \geqslant 0 \text { and } i, j \in \mathbb{N}\right\}, \\
\delta_{n} & =\sup \left\{d\left(x_{i}, x_{j}\right): i, j \geqslant n \text { and } i, j \in \mathbb{N}\right\},
\end{aligned}
$$

for all $n \in \mathbb{N}$. The proof will be broken into six steps.

Step 1. We prove

$$
\lim _{n \rightarrow \infty} d\left(x_{n}, x_{n+k}\right)=0, k=2,3, \cdots, p .
$$

Using (2.2) and the triangular inequality, for $k=2,3, \cdots, p$, we have

$$
d\left(x_{n}, x_{n+k}\right) \leqslant s d\left(x_{n}, x_{n+1}\right)+s^{2} d\left(x_{n+1}, x_{n+2}\right)+\cdots+s^{k} d\left(x_{n+k-1}, x_{n+k}\right),
$$

implies

$$
\lim _{n \rightarrow \infty} d\left(x_{n}, x_{n+k}\right)=0 .
$$

Further, we see the set $\left\{d\left(x_{n}, x_{n+k}\right): k=1,2, \cdots, p\right.$ and $\left.n=0,1,2, \cdots\right\}$ is bounded, that is, there exists $M>0$ such that

$$
d\left(x_{n}, x_{n+k}\right) \leqslant M
$$

for all $k \in\{1,2, \cdots, p\}$ and $n \in\{0,1,2, \cdots\}$.

We put

$$
\eta_{n}=\max \left\{d\left(x_{n}, x_{n+k}\right): k=1,2, \cdots, p\right\},
$$

for $n \in \mathbb{N}$. Then we deduce that $\lim _{n \rightarrow \infty} \eta_{n}=0$ and

$$
\lim _{n \rightarrow \infty}\left(\sup \left\{\eta_{i}: i \geqslant n\right\}\right)=0 .
$$

Step 2. We prove $\left\{D_{n}\right\}$ is bounded and $D_{\infty}<\infty$.

Let $n \in \mathbb{N}$ be given. For any $i, j \in \mathbb{N}$ with $0 \leqslant i, j \leqslant n$ and $i \neq j$, we consider the following three cases.

Case $i$. If $1 \leqslant i, j \leqslant n$ and $|i-j| \equiv 1 \bmod p$, then by using (2.1), we get

$$
\begin{aligned}
d\left(x_{i}, x_{j}\right) & =d\left(T x_{i-1}, T x_{j-1}\right) \\
& \leqslant \lambda \max \left\{d\left(x_{i-1}, x_{j-1}\right), d\left(x_{i-1}, T x_{i-1}\right), d\left(x_{j-1}, T x_{j-1}\right), d\left(x_{i-1}, T x_{j-1}\right), d\left(x_{j-1}, T x_{i-1}\right)\right\} \\
& =\lambda \max \left\{d\left(x_{i-1}, x_{j-1}\right), d\left(x_{i-1}, x_{i}\right), d\left(x_{j-1}, x_{j}\right), d\left(x_{i-1}, x_{j}\right), d\left(x_{j-1}, x_{i}\right)\right\} .
\end{aligned}
$$

It follows that

$$
d\left(x_{i}, x_{j}\right) \leqslant \lambda D_{n}
$$


Case ii. If $i=0$ and $j \equiv 1 \bmod p$ or $j \equiv(p-1) \bmod p$, then $|p-j| \equiv 1 \bmod p$. Using (2.3) and (2.6), we get

$$
\begin{aligned}
d\left(x_{i}, x_{j}\right) & =d\left(x_{0}, x_{j}\right) \\
& \leqslant s d\left(x_{0}, x_{p}\right)+s d\left(x_{p}, x_{j}\right) \\
& \leqslant s M+s \lambda D_{n} .
\end{aligned}
$$

Case iii. If $0 \leqslant i<j \leqslant n$ and $j-i \not \equiv 1 \bmod p$, then there exists a natural number $t \in\{1,2, \cdots, p-1\}$ such that $j-(i+t) \equiv 1 \bmod p$. Using (2.3) and (2.6), we have

$$
\begin{aligned}
d\left(x_{i}, x_{j}\right) & \leqslant s d\left(x_{i}, x_{i+t}\right)+s d\left(x_{i+t}, x_{j}\right) \\
& \leqslant s M+s \lambda D_{n} .
\end{aligned}
$$

Thus we conclude that

$$
d\left(x_{i}, x_{j}\right) \leqslant s M+s \lambda D_{n}
$$

for all $i, j \in \mathbb{N}$ with $0 \leqslant i, j \leqslant n$, which implies

$$
D_{n} \leqslant s M+s \lambda D_{n} .
$$

Consequently, we see that

$$
D_{n} \leqslant \frac{s M}{1-s \lambda} .
$$

This means that $\left\{D_{n}\right\}$ is bounded and $D_{\infty} \leqslant \frac{s M}{1-s \lambda}<\infty$.

Step 3. We prove

$$
\lim _{n \rightarrow \infty} \delta_{n}=0 .
$$

Since $D_{\infty}<\infty$, we have $\delta_{n} \leqslant \delta_{0}=D_{\infty}<\infty$, for all $n \in \mathbb{N}$. Let $n>1$ be given. For any $i, j \geqslant n$ with $i<j$, we consider the following two cases.

Case i. If $j-i \equiv 1 \bmod p$, then by using (2.1), we get

$$
\begin{aligned}
d\left(x_{i}, x_{j}\right) & =d\left(T x_{i-1}, T x_{j-1}\right) \\
& \leqslant \lambda \max \left\{d\left(x_{i-1}, x_{j-1}\right), d\left(x_{i-1}, T x_{i-1}\right), d\left(x_{j-1}, T x_{j-1}\right), d\left(x_{i-1}, T x_{j-1}\right), d\left(x_{j-1}, T x_{i-1}\right)\right\} \\
& =\lambda \max \left\{d\left(x_{i-1}, x_{j-1}\right), d\left(x_{i-1}, x_{i}\right), d\left(x_{j-1}, x_{j}\right), d\left(x_{i-1}, x_{j}\right), d\left(x_{j-1}, x_{i}\right)\right\} .
\end{aligned}
$$

It follows that

$$
d\left(x_{i}, x_{j}\right) \leqslant \lambda \delta_{n-1},
$$

for all $i, j \geqslant n$ with $j>i$ and $j-i \equiv 1 \bmod p$.

Observe that $|j-(i+p)| \equiv 1 \bmod p$ and $j, i+p \geqslant n+1$. Then by using (2.8), we get

$$
\begin{aligned}
d\left(x_{i}, x_{j}\right) & \leqslant s d\left(x_{i}, x_{i+p}\right)+s d\left(x_{i+p}, x_{j}\right) \\
& \leqslant s d\left(x_{i}, x_{i+p}\right)+s \lambda \delta_{n} .
\end{aligned}
$$

From (2.4), we see that

$$
d\left(x_{i}, x_{j}\right) \leqslant s \eta_{i}+s \lambda \delta_{n} \leqslant \sup \left\{\eta_{i}: i \geqslant n\right\}+s \lambda \delta_{n} .
$$

Case ii. If $j-i \not \equiv 1 \bmod p$, then there exists a natural number $t \in\{1,2, \cdots, p-1\}$ such that $j-(i+t) \equiv 1$ mod p. Using (2.4), (2.8) and $j, i+t \geqslant n+1$, we get that

$$
\begin{aligned}
d\left(x_{i}, x_{j}\right) & \leqslant s d\left(x_{i}, x_{i+t}\right)+s d\left(x_{i+t}, x_{j}\right) \\
& \leqslant s \eta_{i}+s \lambda \delta_{n}
\end{aligned}
$$




$$
\leqslant s \sup \left\{\eta_{i}: i \geqslant n\right\}+s \lambda \delta_{n}
$$

Thus we obtain that

$$
d\left(x_{i}, x_{j}\right) \leqslant s \sup \left\{\eta_{i}: i \geqslant n\right\}+s \lambda \delta_{n},
$$

for all $i, j \geqslant n$. This means that

$$
\delta_{n} \leqslant \sup \left\{\eta_{i}: i \geqslant n\right\}+s \lambda \delta_{n}
$$

which implies

$$
\delta_{n} \leqslant \frac{s}{1-s \lambda} \sup \left\{\eta_{i}: i \geqslant n\right\}
$$

Using (2.5), we get $\lim _{n \rightarrow \infty} \delta_{n}=0$.

Step 4 . We prove $\left\{x_{n}\right\}$ is a b-Cauchy sequence.

For all $n, m \geqslant 1$ with $n<m$, we have $d\left(x_{n}, x_{m}\right) \leqslant \delta_{n}$. From (2.7), it follows that $\left\{x_{n}\right\}$ is a b-Cauchy sequence. Since $X$ is $b$-complete, there exists $x^{*} \in X$ such that

$$
\lim _{n \rightarrow \infty} x_{n}=x^{*}
$$

Using the cyclic character of $T$, there exists a subsequence of $\left\{x_{n}\right\}$ for which belongs to $A_{i}$ for $i \in$ $\{1,2, \cdots, p\}$. Hence, from the closedness of $A_{i}$, we see that $x^{*} \in \cap_{i=1}^{p} A_{i}$.

Step 5 . We prove $x^{*}$ is a fixed point of $T$.

We claim $\lim _{n \rightarrow \infty} x_{n}=T x^{*}$. In fact, by using (2.1), we have

$$
\begin{aligned}
d\left(x_{n+1}, T x^{*}\right) & =d\left(T x_{n}, T x^{*}\right) \\
& \leqslant \lambda \max \left\{d\left(x_{n}, x^{*}\right), d\left(x_{n}, T x_{n}\right), d\left(x^{*}, T x^{*}\right), d\left(x^{*}, T x_{n}\right), d\left(x_{n}, T x^{*}\right)\right\} \\
& =\lambda \max \left\{d\left(x_{n}, x^{*}\right), d\left(x_{n}, x_{n+1}\right), d\left(x^{*}, T x^{*}\right), d\left(x^{*}, x_{n+1}\right), d\left(x_{n}, T x^{*}\right)\right\} .
\end{aligned}
$$

Notice the following two facts.

(1) If $d\left(x_{n+1}, T x^{*}\right) \leqslant \lambda d\left(x^{*}, T x^{*}\right)$, then we have $d\left(x_{n+1}, T x^{*}\right) \leqslant s \lambda d\left(x^{*}, x_{n+1}\right)+s \lambda d\left(x_{n+1}, T x^{*}\right)$, which implies

$$
d\left(x_{n+1}, T x^{*}\right) \leqslant \frac{s \lambda}{1-s \lambda} d\left(x^{*}, x_{n+1}\right) .
$$

(2) If $d\left(x_{n+1}, T x^{*}\right) \leqslant \lambda d\left(x_{n}, T x^{*}\right)$, then we have $d\left(x_{n+1}, T x^{*}\right) \leqslant s \lambda d\left(x_{n}, x_{n+1}\right)+s \lambda d\left(x_{n+1}, T x^{*}\right)$, which implies

$$
d\left(x_{n+1}, T x^{*}\right) \leqslant \frac{s \lambda}{1-s \lambda} d\left(x_{n}, x_{n+1}\right)
$$

Thus

$$
\begin{aligned}
d\left(x_{n+1}, T x^{*}\right) & \leqslant \max \left\{\lambda d\left(x_{n}, x^{*}\right), \lambda d\left(x_{n}, x_{n+1}\right), \frac{s \lambda}{1-s \lambda} d\left(x^{*}, x_{n+1}\right), \lambda d\left(x^{*}, x_{n+1}\right), \frac{s \lambda}{1-s \lambda} d\left(x_{n}, x_{n+1}\right)\right\} \\
& \leqslant \frac{s \lambda}{1-s \lambda} \max \left\{d\left(x_{n}, x^{*}\right), d\left(x_{n}, x_{n+1}\right), d\left(x^{*}, x_{n+1}\right)\right\} .
\end{aligned}
$$

Using (2.2) and (2.9), we see that $\lim _{n \rightarrow \infty} x_{n}=T x^{*}$. Since a b-convergent sequence has a unique limit in b-metric spaces, we get $T x^{*}=x^{*}$, that is $x^{*}$ is a fixed point of $T$.

Step 6. We prove the fixed point of $\mathrm{T}$ is unique.

Suppose that $\mathrm{y}^{*}$ is another fixed point of T. Then using (2.1), we have

$$
\begin{aligned}
d\left(x^{*}, y^{*}\right) & =d\left(T x^{*}, T y^{*}\right) \\
& \leqslant \lambda \max \left\{d\left(x^{*}, y^{*}\right), d\left(x^{*}, T x^{*}\right), d\left(y^{*}, T y^{*}\right), d\left(x^{*}, T y^{*}\right), d\left(y^{*}, T x^{*}\right)\right\} \\
& =\lambda d\left(x^{*}, y^{*}\right) .
\end{aligned}
$$

Since $\lambda<\frac{1}{s} \leqslant 1$, we deduce that $d\left(x^{*}, y^{*}\right)=0$ and $x^{*}=y^{*}$. 
Remark 2.5. If we take $A_{i}=X$, for all $1 \leqslant i \leqslant p$, in Lemma 2.4, we can get that the non-cyclic case of Lemma 2.4.

Theorem 2.6. Let $(X, d)$ be a b-complete b-metric space with $s \geqslant 1$ and $\left\{A_{i}\right\}_{i=1}^{p}$ nonempty closed subsets of $X$. Suppose that $T: \cup_{i=1}^{p} A_{i} \rightarrow \cup_{i=1}^{p} A_{i}$ satisfies the following conditions (where $A_{p+1}=A_{1}$ )

(i) $\mathrm{T}\left(A_{i}\right) \subseteq A_{i+1}$, for $1 \leqslant i \leqslant p$;

(ii) there exists $\lambda \in\left[0, \frac{1}{s}\right)$ such that

$$
d(T x, T y) \leqslant \lambda \max \left\{d(x, y), d(x, T x), d(y, T y), \frac{d(x, T y)+d(y, T x)}{2}\right\},
$$

for all $x \in A_{i}, y \in A_{i+1}, 1 \leqslant i \leqslant p$.

Then $\mathrm{T}$ has a unique fixed point.

Proof. Since the condition (2.10) implies the condition (2.1), it suffices to prove the condition (F) in Lemma 2.4 holds. Let $x_{0} \in \cup_{i=1}^{p} A_{i}$ be given and let $x_{n}=T x_{n-1}, n=1,2, \cdots$. Using (2.10),

$$
\begin{aligned}
d\left(x_{n}, x_{n+1}\right) & \leqslant \lambda \max \left\{d\left(x_{n-1}, x_{n}\right), d\left(x_{n-1}, x_{n}\right), d\left(x_{n}, x_{n+1}\right), \frac{d\left(x_{n-1}, x_{n+1}\right)+d\left(x_{n}, x_{n}\right)}{2}\right\} \\
& =\lambda \max \left\{d\left(x_{n-1}, x_{n}\right), d\left(x_{n}, x_{n+1}\right), \frac{d\left(x_{n-1}, x_{n+1}\right)}{2}\right\} .
\end{aligned}
$$

We get the following three cases.

Case i. If $\max \left\{d\left(x_{n-1}, x_{n}\right), d\left(x_{n}, x_{n+1}\right), \frac{d\left(x_{n-1}, x_{n+1}\right)}{2}\right\}=d\left(x_{n-1}, x_{n}\right)$, then $d\left(x_{n}, x_{n+1}\right) \leqslant \lambda d\left(x_{n-1}, x_{n}\right)$.

Case ii. If $\max \left\{d\left(x_{n-1}, x_{n}\right), d\left(x_{n}, x_{n+1}\right), \frac{d\left(x_{n-1}, x_{n+1}\right)}{2}\right\}=d\left(x_{n}, x_{n+1}\right)$, then $d\left(x_{n}, x_{n+1}\right) \leqslant \lambda d\left(x_{n}, x_{n+1}\right)$. From $\lambda<1$, we see that $d\left(x_{n}, x_{n+1}\right)=0$.

Case iii. If $\max \left\{d\left(x_{n-1}, x_{n}\right), d\left(x_{n}, x_{n+1}\right), \frac{d\left(x_{n-1}, x_{n+1}\right)}{2}\right\}=\frac{d\left(x_{n-1}, x_{n+1}\right)}{2}$, then

$$
\begin{aligned}
d\left(x_{n}, x_{n+1}\right) & \leqslant \lambda \cdot \frac{d\left(x_{n-1}, x_{n+1}\right)}{2} \\
& \leqslant \frac{s \lambda}{2}\left[d\left(x_{n-1}, x_{n}\right)+d\left(x_{n}, x_{n+1}\right)\right],
\end{aligned}
$$

which implies

$$
d\left(x_{n}, x_{n+1}\right) \leqslant \frac{s \lambda}{2-s \lambda} d\left(x_{n-1}, x_{n}\right)
$$

Put $\beta=\max \left\{\frac{s \lambda}{2-s \lambda}, \lambda\right\}$. Then we conclude that

$$
d\left(x_{n}, x_{n+1}\right) \leqslant \beta d\left(x_{n-1}, x_{n}\right),
$$

for all $n \in \mathbb{N}$. This leads to

$$
d\left(x_{n}, x_{n+1}\right) \leqslant \beta^{n} d\left(x_{0}, x_{1}\right) .
$$

Since $\beta<1$, we get $\lim _{n \rightarrow \infty} d\left(x_{n}, x_{n+1}\right)=0$, that is the condition (F) holds.

Remark 2.7. If we replace the condition (2.10) in Theorem 2.6 by the following stronger condition

$$
d(T x, T y) \leqslant \lambda \max \left\{d(x, y), d(x, T x), d(y, T y), \frac{d(x, T y)+d(y, T x)}{2 s}\right\},
$$

then the proof of Theorem 2.6 will become straightforward because we can prove

$$
d\left(x_{n}, x_{n+1}\right) \leqslant \lambda^{n} d\left(x_{0}, x_{1}\right),
$$

which implies that $\left\{x_{n}\right\}$ is b-Cauchy. The following example shows that the condition (2.11) is strict stronger than the condition (2.10). 
Example 2.8. Let $X=\mathbb{R}$ and let $d(x, y)=(x-y)^{2}$ for each $x, y \in X$. Then $(X, d)$ is a b-complete b-metric space with $s=2$. Set

Define $T: A \cup B \rightarrow A \cup B$ by

$$
A=\{0,1\}, B=\left\{0, \frac{11}{5}\right\}
$$

$$
\mathrm{T} 0=\mathrm{T} 1=0, \quad \mathrm{~T} \frac{11}{5}=1 .
$$

We have $T A \subseteq B$ and $T B \subseteq A$. Further, we have

$$
\begin{aligned}
d(T 0, T 0) & =d(T 0, T 1)=0, \\
d\left(T 0, T \frac{11}{5}\right) & =d(0,1)=1<\frac{1}{2} d\left(0, \frac{11}{5}\right)=\frac{121}{50}, \\
d\left(T 1, T \frac{11}{5}\right) & =d(0,1)=1<\frac{1}{2} \frac{d\left(1, T \frac{11}{5}\right)+d\left(\frac{11}{5}, T 1\right)}{2}=\frac{121}{100} .
\end{aligned}
$$

Thus $T$ satisfies all conditions of Theorem 2.6. In fact, 0 is the unique fixed point of $T$. But $T$ does not satisfy the condition (2.11) because

$$
\begin{aligned}
\mathrm{d}\left(\mathrm{T} 1, \mathrm{~T} \frac{11}{5}\right) & =\mathrm{d}(0,1)=1 \\
& >\frac{1}{2} \max \left\{\mathrm{d}\left(1, \frac{11}{5}\right), \mathrm{d}(1, \mathrm{~T} 1), \mathrm{d}\left(\frac{11}{5}, \mathrm{~T} \frac{11}{5}\right), \frac{\mathrm{d}\left(1, \mathrm{~T} \frac{11}{5}\right)+\mathrm{d}\left(\frac{11}{5}, \mathrm{~T} 1\right)}{2 \cdot 2}\right\} \\
& =\frac{1}{2} \max \left\{\frac{36}{25}, 1, \frac{36}{25}, \frac{121}{100}\right\}=\frac{18}{25} .
\end{aligned}
$$

Theorem 2.9. Let $(X, d)$ be a b-complete b-metric space with $s \geqslant 1$ and $\left\{A_{i}\right\}_{i=1}^{p}$ nonempty closed subsets of $X$. Suppose that $\mathrm{T}: \cup_{i=1}^{p} A_{i} \rightarrow \cup_{i=1}^{p} A_{i}$ satisfies the following conditions (where $A_{p+1}=A_{1}$ )

(i) $\mathrm{T}\left(A_{i}\right) \subseteq A_{i+1}$ for $1 \leqslant i \leqslant p$;

(ii) there exists $\lambda \in\left[0, \frac{1}{\mathrm{~s}}\right.$ ) such that

$$
d(T x, T y) \leqslant \lambda \max \left\{d(x, y), d(x, T x), d(y, T y), \frac{d(x, T y)}{2}, d(y, T x)\right\}
$$

for all $x \in A_{i}, y \in A_{i+1}, 1 \leqslant i \leqslant p$.

Then $\mathrm{T}$ has a unique fixed point.

Proof. Since the condition (2.12) implies the condition (2.1), it suffices to prove the condition (F) in Lemma 2.4 holds. Let $x_{0} \in \cup_{i=1}^{p} A_{i}$ be given and let $x_{n}=T x_{n-1}, n=1,2, \cdots$. Using (2.12),

$$
\begin{aligned}
d\left(x_{n}, x_{n+1}\right) & =d\left(T x_{n-1}, T x_{n}\right) \\
& \leqslant \lambda \max \left\{d\left(x_{n-1}, x_{n}\right), d\left(x_{n-1}, x_{n}\right), d\left(x_{n}, x_{n+1}\right), \frac{d\left(x_{n-1}, x_{n+1}\right)}{2}, d\left(x_{n}, x_{n}\right)\right\} \\
& =\lambda \max \left\{d\left(x_{n-1}, x_{n}\right), d\left(x_{n}, x_{n+1}\right), \frac{d\left(x_{n-1}, x_{n+1}\right)}{2}\right\} .
\end{aligned}
$$

Similar to the proof in Theorem 2.6, we can get the condition (F) holds.

The next are two examples which show that Theorem 2.6 and Theorem 2.9 are independent of each other.

Example 2.10. Let $X=\mathbb{R}$ and let $d(x, y)=(x-y)^{2}$ for each $x, y \in X$. Then $(X, d)$ is a b-complete b-metric space with $s=2$. Set

$$
A=\left\{0,-\frac{1}{5}\right\}, \quad B=\{0,1,2\}, \quad C=\left\{0, \frac{9}{10}, \frac{4}{5}\right\} .
$$


Define $T: A \cup B \cup C \rightarrow A \cup B \cup C$ by

$$
\begin{aligned}
\mathrm{T} 0 & =\mathrm{T}\left(-\frac{1}{5}\right)=\mathrm{T} 1=\mathrm{T} \frac{9}{10}=0, \\
\mathrm{~T} 2 & =\frac{9}{10}, \quad \mathrm{~T} \frac{4}{5}=-\frac{1}{5} .
\end{aligned}
$$

We have $\mathrm{TA} \subseteq \mathrm{B}, \mathrm{TB} \subseteq \mathrm{C}$ and $\mathrm{TC} \subseteq \mathrm{A}$. Further, we have

$$
\begin{aligned}
& \mathrm{d}(\mathrm{T} 0, \mathrm{~T} 0)=\mathrm{d}(\mathrm{T} 0, \mathrm{~T} 1)=0, \\
& \mathrm{~d}(\mathrm{~T} 0, \mathrm{~T} 2)=\mathrm{d}\left(0, \frac{9}{10}\right)=\frac{81}{100}<\frac{1}{2} \mathrm{~d}(0,2)=2, \\
& \mathrm{~d}\left(\mathrm{~T}\left(-\frac{1}{5}\right), \mathrm{T} 0\right)=\mathrm{d}\left(\mathrm{T}\left(-\frac{1}{5}\right), \mathrm{T} 1\right)=0, \\
& \mathrm{~d}\left(\mathrm{~T}\left(-\frac{1}{5}\right), \mathrm{T} 2\right)=\mathrm{d}\left(0, \frac{9}{10}\right)=\frac{81}{100}<\frac{1}{2} \mathrm{~d}\left(\left(-\frac{1}{5}\right), 2\right)=\frac{121}{50}, \\
& \mathrm{~d}\left(\mathrm{~T} 0, \mathrm{~T} \frac{9}{10}\right)=\mathrm{d}\left(\mathrm{T} 1, \mathrm{~T} \frac{9}{10}\right)=0, \\
& \mathrm{~d}\left(\mathrm{~T} 0, \mathrm{~T} \frac{4}{5}\right)=\mathrm{d}\left(0,-\frac{1}{5}\right)=\frac{1}{25}<\frac{1}{2} \mathrm{~d}\left(0, \frac{4}{5}\right)=\frac{8}{25}, \\
& \mathrm{~d}\left(\mathrm{~T} 1, \mathrm{~T} \frac{4}{5}\right)=\mathrm{d}\left(0,-\frac{1}{5}\right)=\frac{1}{25}<\frac{1}{2} \mathrm{~d}(1, \mathrm{~T} 1)=\frac{1}{2}, \\
& \mathrm{~d}(\mathrm{~T} 2, \mathrm{~T} 0)=\mathrm{d}\left(\frac{9}{10}, 0\right)=\frac{81}{100}<\frac{1}{2} \mathrm{~d}(2,0)=\frac{1}{2}, \\
& \mathrm{~d}\left(\mathrm{~T} 2, \mathrm{~T} \frac{9}{10}\right)=\mathrm{d}\left(\frac{9}{10}, 0\right)=\frac{81}{100}<\frac{1}{2} \frac{\mathrm{d}\left(2, \mathrm{~T} \frac{9}{10}\right)+\mathrm{d}\left(\frac{9}{10}, \mathrm{~T} 2\right)}{2}=1 \text {, } \\
& \mathrm{d}\left(\mathrm{T} 2, \mathrm{~T} \frac{4}{5}\right)=\mathrm{d}\left(\frac{9}{10},-\frac{1}{5}\right)=\frac{121}{100}<\frac{1}{2} \frac{\mathrm{d}\left(2, \mathrm{~T} \frac{4}{5}\right)+\mathrm{d}\left(\frac{4}{5}, \mathrm{~T} 2\right)}{2}=\frac{485}{400}, \\
& \mathrm{~d}\left(\mathrm{~T} 0, \mathrm{~T}\left(-\frac{1}{5}\right)\right)=\mathrm{d}\left(\mathrm{T} \frac{9}{10}, \mathrm{~T} 0\right)=\mathrm{d}\left(\mathrm{T} \frac{9}{10}, \mathrm{~T}\left(-\frac{1}{5}\right)\right)=0 \text {, } \\
& \mathrm{d}\left(\mathrm{T} \frac{4}{5}, \mathrm{~T} 0\right)=\mathrm{d}\left(-\frac{1}{5}, 0\right)=\frac{1}{25}<\frac{1}{2} \mathrm{~d}\left(\frac{4}{5}, 0\right)=\frac{8}{25}, \\
& \mathrm{~d}\left(\mathrm{~T} \frac{4}{5}, \mathrm{~T}\left(-\frac{1}{5}\right)\right)=\mathrm{d}\left(-\frac{1}{5}, 0\right)=\frac{1}{25}<\frac{1}{2} \mathrm{~d}\left(\frac{4}{5},-\frac{1}{5}\right)=\frac{1}{2} \text {. }
\end{aligned}
$$

Thus $T$ satisfies all conditions of Theorem 2.6. In fact, 0 is the unique fixed point of $T$. But $T$ does not satisfy the condition (2.12) in Theorem 2.9 because

$$
\begin{aligned}
\mathrm{d}\left(\mathrm{T} 2, \mathrm{~T} \frac{4}{5}\right) & =\mathrm{d}\left(\frac{9}{10},-\frac{1}{5}\right)=\frac{121}{100} \\
& =\frac{1}{2} \max \left\{\mathrm{d}\left(2, \frac{4}{5}\right), \mathrm{d}(2, \mathrm{~T} 2), \mathrm{d}\left(\frac{4}{5}, \mathrm{~T} \frac{4}{5}\right), \frac{1}{2} \mathrm{~d}\left(2, \mathrm{~T} \frac{4}{5}\right), \mathrm{d}\left(\frac{4}{5}, \mathrm{~T} 2\right)\right\} \\
& =\frac{1}{2} \max \left\{\frac{36}{25}, \frac{121}{100}, 1, \frac{121}{50}, \frac{1}{100}\right\} .
\end{aligned}
$$

Example 2.11. Let $X=\mathbb{R}$ and let $d(x, y)=(x-y)^{2}$ for each $x, y \in X$. Then $(X, d)$ is a b-complete b-metric space with $s=2$. Set

$$
A=\left\{0, \frac{9}{20}, 2\right\}, B=\left\{0, \frac{9}{10}, \frac{37}{20}\right\}, C=\{0,1\} .
$$

Define $T: A \cup B \cup C \rightarrow A \cup B \cup C$ by

$$
\mathrm{T} 0=\mathrm{T} \frac{9}{20}=\mathrm{T} \frac{9}{10}=0,
$$




$$
\mathrm{T} 2=\frac{9}{10}, \quad \mathrm{~T} \frac{37}{20}=1, \quad \mathrm{~T} 1=\frac{9}{20} .
$$

We have $\mathrm{TA} \subseteq \mathrm{B}, \mathrm{TB} \subseteq \mathrm{C}$ and $\mathrm{TC} \subseteq \mathrm{A}$. Further, we have

$$
\begin{aligned}
& \mathrm{d}(\mathrm{T} 0, \mathrm{~T} 0)=\mathrm{d}\left(\mathrm{T} 0, \mathrm{~T} \frac{9}{10}\right)=0, \\
& \mathrm{~d}\left(\mathrm{~T} 0, \mathrm{~T} \frac{37}{20}\right)=\mathrm{d}(0,1)=1<\frac{1}{2} \mathrm{~d}\left(0, \frac{37}{20}\right)=\frac{1369}{800} \text {, } \\
& \mathrm{d}\left(\mathrm{T} \frac{9}{20}, \mathrm{~T} 0\right)=\mathrm{d}\left(\mathrm{T} \frac{9}{20}, \mathrm{~T} \frac{9}{10}\right)=0 \text {, } \\
& \mathrm{d}\left(\mathrm{T} \frac{9}{20}, \mathrm{~T} \frac{37}{20}\right)=\mathrm{d}(0,1)=1<\frac{1}{2} \mathrm{~d}\left(\frac{37}{20}, \mathrm{~T} \frac{9}{20}\right)=\frac{1369}{800} \text {, } \\
& \mathrm{d}(\mathrm{T} 2, \mathrm{~T} 0)=\mathrm{d}\left(\frac{9}{10}, 0\right)=\frac{81}{100}<\frac{1}{2} \mathrm{~d}(2,0)=2, \\
& \mathrm{~d}\left(\mathrm{~T} 2, \mathrm{~T} \frac{9}{10}\right)=\mathrm{d}\left(\frac{9}{10}, 0\right)=\frac{81}{100}<\frac{1}{2} \cdot \frac{1}{2} \mathrm{~d}\left(2, \mathrm{~T} \frac{9}{10}\right)=1 \text {, } \\
& \mathrm{d}\left(\mathrm{T} 2, \mathrm{~T} \frac{37}{20}\right)=\mathrm{d}\left(\frac{9}{10}, 1\right)=\frac{1}{100}<\frac{1}{2} \mathrm{~d}\left(2, \frac{37}{20}\right)=\frac{9}{800} \text { ， } \\
& \mathrm{d}(\mathrm{T} 0, \mathrm{~T} 1)=\mathrm{d}\left(0, \frac{9}{20}\right)=\frac{81}{400}<\frac{1}{2} \mathrm{~d}(0,1)=\frac{1}{2}, \\
& \mathrm{~d}\left(\mathrm{~T} \frac{9}{10}, \mathrm{~T} 0\right)=0 \text {, } \\
& \mathrm{d}\left(\mathrm{T} \frac{9}{10}, \mathrm{~T} 1\right)=\mathrm{d}\left(0, \frac{9}{20}\right)=\frac{81}{400}<\frac{1}{2} \mathrm{~d}\left(\frac{9}{10}, \mathrm{~T} \frac{9}{10}\right)=\frac{81}{200} ， \\
& \mathrm{~d}\left(\mathrm{~T} \frac{37}{20}, \mathrm{~T} 0\right)=\mathrm{d}(1,0)=1<\frac{1}{2} \mathrm{~d}\left(\frac{37}{20}, 0\right)=\frac{1369}{800}, \\
& \mathrm{~d}\left(\mathrm{~T} \frac{37}{20}, \mathrm{~T} 1\right)=\mathrm{d}\left(1, \frac{9}{20}\right)=\frac{121}{400}<\frac{1}{2} \mathrm{~d}\left(\frac{37}{20}, 1\right)=\frac{289}{800} \text {, } \\
& \mathrm{d}\left(\mathrm{T} 0, \mathrm{~T} \frac{9}{20}\right)=0 \text {, } \\
& \mathrm{d}(\mathrm{T} 0, \mathrm{~T} 2)=\mathrm{d}\left(0, \frac{9}{10}\right)=\frac{81}{100}<\frac{1}{2} \mathrm{~d}(0,2)=2, \\
& \mathrm{~d}(\mathrm{~T} 1, \mathrm{~T} 0)=\mathrm{d}\left(\frac{9}{20}, 0\right)=\frac{81}{400}<\frac{1}{2} \mathrm{~d}(1,0)=\frac{1}{2}, \\
& \mathrm{~d}\left(\mathrm{~T} 1, \mathrm{~T} \frac{9}{20}\right)=\mathrm{d}\left(\frac{9}{20}, 0\right)=\frac{81}{400}<\frac{1}{2} \cdot \frac{1}{2} \mathrm{~d}\left(1, \mathrm{~T} \frac{9}{20}\right)=\frac{1}{4}, \\
& \mathrm{~d}(\mathrm{~T} 1, \mathrm{~T} 2)=\mathrm{d}\left(\frac{9}{20}, \frac{9}{10}\right)=\frac{81}{400}<\frac{1}{2} \mathrm{~d}(1,2)=\frac{1}{2} .
\end{aligned}
$$

Thus $T$ satisfies all conditions of Theorem 2.9. In fact, 0 is the unique fixed point of $T$. But $T$ does not satisfy the condition (2.10) in Theorem 2.6 because

$$
\begin{aligned}
\mathrm{d}\left(\mathrm{T} \frac{9}{20}, \mathrm{~T} \frac{37}{20}\right) & =\mathrm{d}(0,1)=1 \\
& >\frac{1}{2} \max \left\{\mathrm{d}\left(\frac{9}{20}, \frac{37}{20}\right), \mathrm{d}\left(\frac{9}{20}, \mathrm{~T} \frac{9}{20}\right), \mathrm{d}\left(\frac{37}{20}, \mathrm{~T} \frac{37}{20}\right), \frac{\mathrm{d}\left(\frac{9}{20}, \mathrm{~T} \frac{37}{20}\right)+\mathrm{d}\left(\frac{37}{20}, \mathrm{~T} \frac{9}{20}\right)}{2}\right\} \\
& =\frac{1}{2} \max \left\{\frac{49}{25}, \frac{81}{400}, \frac{289}{400}, \frac{149}{80}\right\} \\
& =\frac{49}{50} .
\end{aligned}
$$

Now we give a result for non-cyclic quasi-contractive mappings of Ćirić type, which improves the result of Amini-Harandi in [1]. 
Theorem 2.12. Let $(\mathrm{X}, \mathrm{d})$ be $\mathrm{a} \mathrm{b}$-complete $\mathrm{b}$-metric space with $\mathrm{s} \geqslant 1$ and $\mathrm{T}: \mathrm{X} \rightarrow \mathrm{X}$ a self-mapping. Suppose that there exists $\lambda \in\left[0, \frac{1}{s}\right)$ such that

$$
d(T x, T y) \leqslant \lambda \max \{d(x, y), d(x, T x), d(y, T y), d(x, T y), d(y, T x)\},
$$

for all $x, y \in X$. Then $\mathrm{T}$ has a unique fixed point.

Proof. Using the non-cyclic case of Lemma 2.4, we see that it suffices to prove the condition (F) holds. Let $x_{0} \in X$ be given and let $x_{n}=T x_{n-1}, n=1,2, \cdots$. Denote

$$
\begin{gathered}
D_{n}=\max \left\{d\left(x_{i}, x_{j}\right): 0 \leqslant i, j \leqslant n\right\}, \\
\rho_{n}=\max \left\{d\left(x_{0}, x_{j}\right): 1 \leqslant j \leqslant n\right\} .
\end{gathered}
$$

Then we can prove $D_{n}=\rho_{n}$, for any $n \in \mathbb{N}$. Indeed, we easily see that $\rho_{n} \leqslant D_{n}$. Next we show $D_{n} \leqslant \rho_{n}$.

Let $n \in \mathbb{N}$ be given. If $1 \leqslant i, j \leqslant n$, then, using (2.13), we get

$$
\begin{aligned}
d\left(x_{i}, x_{j}\right) & =d\left(T x_{i-1}, T x_{j-1}\right) \\
& \leqslant \lambda \max \left\{d\left(x_{i-1}, x_{j-1}\right), d\left(x_{i-1}, T x_{i-1}\right), d\left(x_{j-1}, T x_{j-1}\right), d\left(x_{i-1}, T x_{j-1}\right), d\left(x_{j-1}, T x_{i-1}\right)\right\} \\
& =\lambda \max \left\{d\left(x_{i-1}, x_{j-1}\right), d\left(x_{i-1}, x_{i}\right), d\left(x_{j-1}, x_{j}\right), d\left(x_{i-1}, x_{j}\right), d\left(x_{j-1}, x_{i}\right)\right\} \\
& \leqslant \lambda D_{n}<D_{n} .
\end{aligned}
$$

Hence $D_{n}=d\left(x_{0}, x_{l}\right)$ for some $1 \leqslant l \leqslant n$. This leads to $D_{n} \leqslant \rho_{n}$.

Now we claim that $\left\{D_{n}\right\}$ is bounded. In fact, for fixed $n \in \mathbb{N}$, by using (2.13), we have

$$
\begin{aligned}
d\left(x_{0}, x_{j}\right) & \leqslant s d\left(x_{0}, x_{1}\right)+s d\left(x_{1}, x_{j}\right) \\
& \leqslant s d\left(x_{0}, x_{1}\right)+s \lambda \max \left\{d\left(x_{0}, x_{j-1}\right), d\left(x_{0}, x_{1}\right) d\left(x_{j-1}, x_{j}\right) d\left(x_{0}, x_{j}\right) d\left(x_{j-1}, x_{1}\right)\right\} \\
& \leqslant s d\left(x_{0}, x_{1}\right)+s \lambda D_{n},
\end{aligned}
$$

for all $j \leqslant n$, which implies that

$$
D_{n}=\rho_{n} \leqslant s d\left(x_{0}, x_{1}\right)+s \lambda D_{n}
$$

Thus

$$
D_{n} \leqslant \frac{s}{1-s \lambda} d\left(x_{0}, x_{1}\right)
$$

that is, $\left\{D_{n}\right\}$ is bounded and

$$
D_{\infty} \triangleq \sup \left\{d\left(x_{i}, x_{j}\right): i, j \geqslant 0\right\} \leqslant \frac{s}{1-s \lambda} d\left(x_{0}, x_{1}\right) .
$$

We put $\delta_{n}=\sup \left\{d\left(x_{i}, x_{j}\right): i, j \geqslant n\right\}$ for $n \in \mathbb{N}$. Then the condition (2.13) implies $\delta_{n} \leqslant \lambda \delta_{n-1}$ for all $n \geqslant 1$. Thus we get

$$
\begin{aligned}
d\left(x_{n}, x_{n+1}\right) & \leqslant \delta_{n} \leqslant \lambda \delta_{n-1} \\
& \leqslant \cdots \leqslant \lambda^{n} \delta_{0} \\
& =\lambda^{n} D_{\infty} \leqslant \frac{s \lambda^{n}}{1-s \lambda} d\left(x_{0}, x_{1}\right) .
\end{aligned}
$$

Since $\lambda<1$, we get $\lim _{n \rightarrow \infty} d\left(x_{n}, x_{n+1}\right)=0$, that is the condition (F) holds.

Remark 2.13. From Theorem 2.12, we see that the condition of Fatou property in the result of AminiHarandi [1] may be omitted.

Following the ideas of Radenović et al. in [16], we see that in Question 1.2, if $\cap_{i=1}^{p} A_{i} \neq \emptyset$ then T must have a fixed point. Now, we will consider that if the intersection of two sets in $\left\{A_{i}\right\}_{i=1}^{p}$ is non-empty then 
whether T have a fixed point. Similar to Example 2.1, we give the following example which shows that $T$ may not have a fixed point if the intersection of two sets in $\left\{A_{i}\right\}_{i=1}^{4}$ is non-empty. Meanwhile we find that if $p=3$ and the intersection of two sets in $\left\{A_{i}\right\}_{i=1}^{p}$ is non-empty then $T$ must have a fixed point.

Example 2.14. Let $X$ be the Euclidean space $\mathbb{R}^{3}$ with the usual metric. Let $A_{1}=\{x, y\}, A_{2}=\{u, v, w\}$, $A_{3}=\{x, y, z\}$ and $A_{4}=\{u, v\}$, where

$$
x=(0,0,0), \quad y=(4,0,0), \quad z=(-4,0,0), \quad u=(2,2,0), \quad v=(2,-2,1), \quad w=(0,4,0) .
$$

Then

$$
\begin{array}{llll}
\mathrm{d}(\mathrm{x}, \mathrm{y})=4, & \mathrm{~d}(\mathrm{x}, \mathrm{u})=2 \sqrt{2}, & \mathrm{~d}(\mathrm{x}, \mathrm{v})=3, & \mathrm{~d}(\mathrm{x}, w)=4, \\
\mathrm{~d}(\mathrm{y}, \mathrm{u})=2 \sqrt{2}, & \mathrm{~d}(\mathrm{y}, \mathrm{v})=3, & \mathrm{~d}(\mathrm{u}, \mathrm{v})=\sqrt{17}, & \mathrm{~d}(\mathrm{y}, w)=4 \sqrt{2}, \\
\mathrm{~d}(\mathrm{z}, \mathrm{u})=2 \sqrt{10}, & \mathrm{~d}(\mathrm{z}, \mathrm{v})=\sqrt{41}, & \mathrm{~d}(\mathrm{z}, w)=4 \sqrt{2} . &
\end{array}
$$

Define $T: \cup_{i=1}^{4} A_{i} \rightarrow \cup_{i=1}^{4} A_{i}$ by

$$
\mathrm{T} x=u, \quad \mathrm{Ty}=v, \mathrm{~T} z=v, \quad \mathrm{Tu}=\mathrm{y}, \mathrm{T} v=x \quad \text { and } \quad \mathrm{T} w=y .
$$

According to the definitions of the sets $A_{i}, 1 \leqslant i \leqslant p$ and the mapping $T$, it follows that $T A_{i} \subseteq A_{i+1}$ for $i=1,2,3,4$, where $A_{5}=A_{1}$. Further, we have

$$
\begin{aligned}
& d(T x, T u)=d(T u, T x)=d(u, y)=2 \sqrt{2}<d(x, T u)=d(x, y)=4, \\
& \mathrm{~d}(\mathrm{~T} x, \mathrm{~T} v)=\mathrm{d}(\mathrm{T} v, \mathrm{~T} x)=\mathrm{d}(\mathrm{u}, \mathrm{x})=2 \sqrt{2}<\mathrm{d}(v, \mathrm{Tx})=\mathrm{d}(v, \mathrm{u})=\sqrt{17}, \\
& \mathrm{~d}(\mathrm{~T} x, \mathrm{~T} w)=\mathrm{d}(\mathrm{T} w, \mathrm{~T} x)=\mathrm{d}(u, y)=2 \sqrt{2}<\mathrm{d}(x, w)=4 \text {, } \\
& \mathrm{d}(\mathrm{Ty}, \mathrm{Tu})=\mathrm{d}(\mathrm{Tu}, \mathrm{Ty})=\mathrm{d}(v, \mathrm{y})=3<\mathrm{d}(\mathrm{u}, \mathrm{Ty})=\mathrm{d}(\mathrm{u}, v)=\sqrt{17}, \\
& d(T y, T v)=d(T v, T y)=d(v, x)=3<d(y, T v)=d(y, x)=4, \\
& \mathrm{~d}(\mathrm{Ty}, \mathrm{Tw})=\mathrm{d}(\mathrm{T} w, \mathrm{Ty})=\mathrm{d}(v, y)=3<\mathrm{d}(\mathrm{y}, w)=4 \sqrt{2} \text {, } \\
& d(T z, T u)=d(T u, T z)=d(v, y)=3<d(z, u)=2 \sqrt{10} \text {, } \\
& \mathrm{d}(\mathrm{T} z, \mathrm{~T} v)=\mathrm{d}(\mathrm{T} v, \mathrm{~T} z)=\mathrm{d}(v, x)=3<\mathrm{d}(z, v)=\sqrt{41} \text {, } \\
& \mathrm{d}(\mathrm{T} w, \mathrm{~T} z)=\mathrm{d}(\mathrm{y}, v)=3<\mathrm{d}(w, z)=4 \sqrt{2} \text {. }
\end{aligned}
$$

Thus T satisfies all conditions of Question 1.2, where $s=1$ and $\lambda=\frac{3}{4}$, but $T$ does not have any fixed point in $\cap_{i=1}^{4} A_{i}$.

Theorem 2.15. Let $(X, d)$ be $a$ b-complete $b$-metric space with $s \geqslant 1$ and $\left\{\mathrm{A}_{i}\right\}_{i=1}^{3}$ nonempty closed subsets of $X$. Suppose that $\mathrm{T}: \cup_{i=1}^{3} A_{i} \rightarrow \cup_{i=1}^{3} A_{i}$ satisfies the following conditions (where $A_{4}=A_{1}$ )

(i) $T\left(A_{i}\right) \subseteq A_{i+1}$ for $1 \leqslant i \leqslant 3$;

(ii) there exists $\lambda \in\left[0, \frac{1}{\mathrm{~s}}\right)$ such that

$$
d(T x, T y) \leqslant \lambda \max \{d(x, y), d(x, T x), d(y, T y), d(x, T y), d(y, T x)\},
$$

for all $x \in A_{i}, y \in A_{i+1}, 1 \leqslant i \leqslant 3$.

Then the following statements are equivalent:

(1) $\cap_{i=1}^{3} A_{i} \neq \emptyset$;

(2) $A_{k} \cap A_{k+1} \neq \emptyset$ for all $k \in\{1,2,3\}$; 
(3) $A_{k} \cap A_{k+1} \neq \emptyset$ for some $k \in\{1,2,3\}$;

(4) $A_{1} \cap A_{2} \neq \emptyset$;

(5) Thas a unique fixed point.

Proof. Using the cyclic character of T, it is easy to verify that the conditions (2), (3) and (4) are equivalent. Following the ideas of Radenović et al. in [16], we see that the conditions (1) and (5) are equivalent. It is clear that the condition (1) implies the condition (4). Thus we only need to prove the condition (4) implies the condition (5).

Assume that $A_{1} \cap A_{2} \neq \emptyset$. Let $x_{0} \in A_{1} \cap A_{2}$ and $x_{n}=T x_{n-1}, n=1,2,3, \cdots$. Since $T\left(A_{1} \cap A_{2}\right) \subseteq$ $A_{2} \cap A_{3}, T\left(A_{2} \cap A_{3}\right) \subseteq A_{2} \cap A_{3}$ and $T\left(A_{3} \cap A_{1}\right) \subseteq A_{1} \cap A_{2}$, we get that $x_{3 k} \in A_{1} \cap A_{2}, x_{3 k+1} \in A_{2} \cap A_{3}$ and $x_{3 k+2} \in A_{3} \cap A_{1}$ for all $k=0,1,2, \cdots$. This means that, for all $i, j \in\{0,1,2, \cdots\}, x_{i}$ and $x_{j}$ lie in different adjacently labeled sets $A_{k}$ and $A_{k+1}$ for certain $k \in\{1,2,3\}$, and therefore they can apply the contractive condition (2.14). In the same way as Theorem 2.12, we see that $x_{0}$ satisfies the condition (F) in Lemma 2.4. Thus $\mathrm{T}$ has a unique fixed point.

Remark 2.16. If we take $A_{1}=A_{2}=A_{3}=X$ in Theorem 2.15, we see that Theorem 2.12 is a special case of Theorem 2.15. Furthermore, it is easy to verify that Theorem 2.15 remains true, if $p \leqslant 5$. Then a natural question arises.

Question 2.17. Does Theorem 2.15 hold if $\mathrm{p}>5$ ?

\section{Acknowledgment}

The authors are thankful to the referees for their valuable comments and suggestions to improve this paper. The authors are supported by the National Natural Science Foundation of China (11561049, 11471236).

\section{References}

[1] A. Amini-Harandi, Fixed point theory for quasi-contraction maps in b-metric spaces, Fixed Point Theory, 15 (2014), 351-358. 1, 1, 2, 2.13

[2] H. Aydi, M. F. Bota, E. Karapınar, S. Mitrović, A fixed point theorem for set-valued quasi-contractions in b-metric spaces, Fixed Point Theory Appl., 2012 (2012), 8 pages. 1

[3] I. A. Bakhtin, The contraction mapping principle in almost metric space, (Russian) Functional analysis, (Russian) Ulyanovsk. Gos. Ped. Inst., Ulyanovsk, 30 (1989), 26-37. 1

[4] L. B. Cirić, A generalization of Banach's contraction principle, Proc. Amer. Math. Soc., 45 (1974), 267-273. 1

[5] L. B. Ćirić, Contractive type non-self mappings on metric spaces of hyperbolic type, J. Math. Anal. Appl., 317 (2006), 28-42. 1

[6] S. Czerwik, Contraction mappings in b-metric spaces, Acta Math. Inform. Univ. Ostraviensis, 1 (1993), 5-11. 1, 1.1, 1

[7] K. M. Das, K. V. Naik, Common fixed-point theorems for commuting maps on a metric space, Proc. Amer. Math. Soc., 77 (1979), 369-373. 1

[8] C. Di Bari, P. Vetro, Nonlinear quasi-contractions of Ćirić type, Fixed Point Theory, 13 (2012), 453-459.

[9] F. He, Common fixed points for nonlinear quasi-contractions of Ćirić type, Abstr. Appl. Anal., 2014 (2014), 9 pages.

[10] F. He, X.-Y. Nan, A unified view on common fixed point theorems for Ćirić quasi-contraction maps, Fixed Point Theory Appl., 2015 (2015), 17 pages. 1

[11] N. Hussain, V. Parvaneh, J. R. Roshan, Z. Kadelburg, Fixed points of cyclic weakly $(\psi, \varphi, \mathrm{L}, \mathrm{A}, \mathrm{B})$-contractive mappings in ordered b-metric spaces with applications, Fixed Point Theory Appl., 2013 (2013), 18 pages. 1

[12] N. Hussain, R. Saadati, R. P. Agrawal, On the topology and wt-distance on metric type spaces, Fixed Point Theory Appl., 2014 (2014), 14 pages. 1, 1

[13] D. Ilić, V. Rakočević, Common fixed points for maps on metric space with w-distance, Appl. Math. Comput., 199 (2008), 599-610. 1

[14] A. A. Mukheimer, $\alpha-\psi$-contractive mappings on b-metric space, J. Comput. Anal. Appl., 18 (2015), 636-644. 1

[15] H. K. Nashine, Z. Kadelburg, Cyclic generalized $\varphi$-contractions in b-metric spaces and an application to integral equations, Filomat, 28 (2014), 2047-2057. 
[16] S. Radenović, T. Došenović, T. A. Lampert, Z. Golubovíc, A note on some recent fixed point results for cyclic contractions in b-metric spaces and an application to integral equations, Appl. Math. Comput., 273 (2016), 155-164. 1, 2, 2

[17] J. R. Roshan, N. Shobkolaei, S. Sedghi, M. Abbas, Common fixed point of four maps in b-metric spaces, Hacet. J. Math. Stat., 43 (2014), 613-624. 1

[18] J.-S. Ume, Fixed point theorems related to Ćirić contraction principle, J. Math. Anal. Appl., 225 (1998), 630-640. 1

[19] F. Vetro, S. Radenović, Nonlinear $\psi$-quasi-contractions of iri-type in partial metric spaces, Appl. Math. Comput., 219 (2012), 1594-1600. 1 